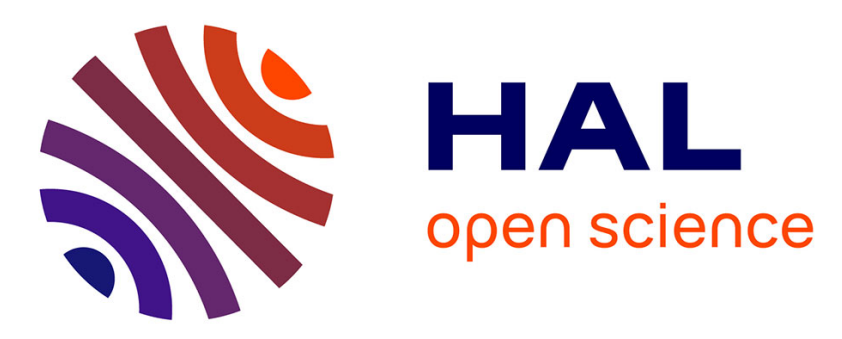

\title{
Experimental Study for Controller-Friendly Contact Estimation for Humanoid Robot
}

Takahiro Ito, Ko Ayusawa, Eiichi Yoshida, Abderrahmane Kheddar

\section{To cite this version:}

Takahiro Ito, Ko Ayusawa, Eiichi Yoshida, Abderrahmane Kheddar. Experimental Study for Controller-Friendly Contact Estimation for Humanoid Robot. ARSO 2019 - 15th IEEE International Conference on Advanced Robotics and its Social Impacts, Oct 2019, Beijing, China. pp.28-33, 10.1109/ARSO46408.2019.8948734 • lirmm-02304148

\section{HAL Id: lirmm-02304148 \\ https://hal-lirmm.ccsd.cnrs.fr/lirmm-02304148}

Submitted on 3 Oct 2019

HAL is a multi-disciplinary open access archive for the deposit and dissemination of scientific research documents, whether they are published or not. The documents may come from teaching and research institutions in France or abroad, or from public or private research centers.
L'archive ouverte pluridisciplinaire HAL, est destinée au dépôt et à la diffusion de documents scientifiques de niveau recherche, publiés ou non, émanant des établissements d'enseignement et de recherche français ou étrangers, des laboratoires publics ou privés. 


\title{
Experimental Study for Controller-Friendly Contact Estimation for Humanoid Robot
}

\author{
Takahiro Ito, Ko Ayusawa, Eiichi Yoshida, and Abderrahmane Kheddar
}

\begin{abstract}
In this paper, we introduce a practical contact observer which allows detecting a contact on the body surface of a humanoid robot. Our method estimates the error force due to undesired contact. Then, contact detection (yes/no) is performed together with the estimation of the contact force and location. By comparing the real robot state according to sensory data with the desired state computed from the contact-free dynamics equation, we can estimate the external force applied to an unknown link. The link in contact is then detected by tracing the torque error along the robot's kinematic chain. Once the link in contact is determined, we can estimate the point where the contact force was applied by using the 3D geometric mesh model of a robot. The proposed method is validated through simulation and experiments using the humanoid robot HRP-4.
\end{abstract}

\section{INTRODUCTION}

Manufacturing in narrow and confined spaces ${ }^{1}$, and assessing and evaluating human assist devices [1], [2], [3], [4], [5] are among the most plausible emerging applications for humanoid robots. In these contexts, when humanoid robots execute the assigned tasks, they are almost always near-tocontact or have to contact with their surroundings. Physical interaction between a humanoid and its surrounding is therefore a critical issue. Physical interaction for manipulation or locomotion requires establishing multiple contacts that must be planned and controlled, considering the stability under any postural situations [6], [7]. While the humanoid must generate necessary contacts to execute its tasks, nondesired contacts may also occur during the motion because of several reasons like errors in modeling and control. These non-desired contacts must be detected and to be handled by the planner or the controller. Several methods have been proposed to detect contacts, as seen in recent research [8], [9], [10], [11], [12]. There are also some works about the physical interaction between a humanoid and a human [13], [14], [15], [16]. When a humanoid robot interacts with a human, contact detection is required to prevent the human from being injured. Detection and estimation of contact force are also important when the humanoid is used to evaluate the supportive torque of assistive exoskeleton devices [17].

When the robot interacts with unknown environment, observing the external force due to the interaction is necessary for compliant behavior. Recent humanoids [18], [19] are

T. Ito and E. Yoshida are with the University of Tsukuba, Japan.

T. Ito, K. Ayusawa, E. Yoshida and A. Kheddar are with CNRS-AIST JRL (Joint Robotics Laboratory), UMI3218/RL, Tsukuba, Japan.

T. Ito and A. Kheddar are also with CNRS-University of Montpellier, LIRMM, Interactive Digital Humans, France.

Corresponding author: T. Ito s1730170es.tsukuba.ac.jp

${ }^{1}$ www. comanoid.eu designed for torque-based control so that they can perform compliant motion at joint level [20]. For position-based controlled robots also, both the force strength and the location of the contact points are required to generate wholebody compliant motion. Some previous research has achieved the contact detection and reaction with fixed-based robot manipulator [21], [22] or wheel-based humanoid [16].

While many contact estimation methods have been proposed, contact detection and contact point estimation has hardly been experimentally demonstrated with real humanoid robots. We therefore propose a practical method for contact estimation using an observer based on a dynamic model and investigate its feasibility with real humanoid hardware. Our contact detection formulation is developed primarily with the perspective to be integrated as observer tasks in our multi-objective task-space controller that is formulated as a quadratic programming (QP) problem [23]. Hence, if we formulate the contact detection as constraints in decision variables similar to that of the controller, it can seamlessly be integrated with the controller. This is also the reason why other methods of contact detection are not considered or compared in this paper. As in [8], [10], [11], the contact estimation on (humanoid) robots is usually achieved by three steps: (1) contact detection, (2) contact force estimation, and (3) contact point localization. Since a humanoid has a floating base, the robot is always in contact with the environment. We thus need to distinguish undesired contact forces from desired ones such as floor reaction forces on feet. Our method basically follows the above three steps. In the contact detection step, the actual generalized forces of the robot are computed by its sensory data, while the desired ones are computed by its dynamics model with compensating the desired generalized forces. The contact detection is performed by monitoring the mismatch between the actual and desired forces. In the contact identification step, the contact force strength is estimated by comparing the measured forces with the expected ones by inverse dynamics computation.

The contact point localization consists of the following two parts: (i) The first part seeks for the link in contact by searching all links along robot kinematic chains from base link to distal links; (ii) The second part estimates the contact point by finding the intersection between the line of action of the contact force and the 3D mesh model (or its convex hull) of the links' covers.

We first introduce our proposed contact observer in Section II, then assess it in simulation and by the experiment of humanoid robot HRP-4 [24] in Section III before concluding 
our paper in Section IV.

\section{Contact Observer on Humanoid Robot}

We propose a contact estimator that provides us contact information including contact detection, contact force identification and contact point localization. In the contact detection and identification part, our estimator determines if there is a contact and computes its strength in terms of force wrench. Then, in the contact point localization part, we estimate the contacted link and the point on the body surface of robot 3D model where the contact occurred.

\section{A. Contact detection and identification}

For humanoids, by observing the forces at their floating body, we can obtain the force acting on the robot. A humanoid dynamic model can be written as:

$$
\boldsymbol{M}(\boldsymbol{q}) \ddot{\boldsymbol{q}}+\boldsymbol{C}(\boldsymbol{q}, \dot{\boldsymbol{q}}) \dot{\boldsymbol{q}}+\boldsymbol{G}(\boldsymbol{q})=\boldsymbol{\tau}_{\boldsymbol{m}}+\sum_{i=1}^{n_{c}} \boldsymbol{J}_{i}^{T} \boldsymbol{F}_{\boldsymbol{i}}+\boldsymbol{\tau}_{\boldsymbol{\delta}}
$$

where $\boldsymbol{q}$ is the generalized joint coordinates consisting of the linear position, the orientation of the floating body or base and the joint angles. $\boldsymbol{M}(\boldsymbol{q})$ is the inertia matrix, $\boldsymbol{C}(\boldsymbol{q}, \dot{\boldsymbol{q}})$ is the matrix including centrifugal and Coriolis effect, $\boldsymbol{G}(\boldsymbol{q})$ is the vector of the gravitational term. $\tau_{m}$ is the vector of joint torque, $\boldsymbol{J}_{i}^{T} \boldsymbol{F}_{\boldsymbol{i}}$ is the desired contact forces which $\boldsymbol{J}$ is the contact Jacobian and $\boldsymbol{F}$ is the contact force measured by force/torque sensors, and $\tau_{\delta}$ is the undesired external contact force that we estimate.

Since base link has no actuated joints, the dynamics equation can be written by the following decomposed form,

$$
\begin{aligned}
\left(\begin{array}{cc}
M_{\boldsymbol{b}}(\boldsymbol{q}) & \boldsymbol{M}_{\boldsymbol{b} \boldsymbol{j}}(\boldsymbol{q}) \\
\boldsymbol{M}_{\boldsymbol{j} \boldsymbol{b}}(\boldsymbol{q}) & \boldsymbol{M}_{\boldsymbol{j}}(\boldsymbol{q})
\end{array}\right)\left(\begin{array}{c}
\ddot{\boldsymbol{q}}_{\boldsymbol{b}} \\
\ddot{\boldsymbol{q}}_{\boldsymbol{j}}
\end{array}\right)+\left(\begin{array}{c}
\boldsymbol{C}_{\boldsymbol{b}}(\boldsymbol{q}, \dot{\boldsymbol{q}}) \\
\boldsymbol{C}_{\boldsymbol{j}}(\boldsymbol{q}, \dot{\boldsymbol{q}})
\end{array}\right)\left(\begin{array}{c}
\dot{\boldsymbol{q}}_{\boldsymbol{b}} \\
\dot{\boldsymbol{q}}_{\boldsymbol{j}}
\end{array}\right)+\left(\begin{array}{c}
\boldsymbol{G}_{\boldsymbol{b}}(\boldsymbol{q}) \\
\boldsymbol{G}_{\boldsymbol{j}}(\boldsymbol{q})
\end{array}\right) \\
=\left(\begin{array}{c}
\mathbf{0} \\
\boldsymbol{\tau}_{\boldsymbol{m}}
\end{array}\right)+\sum_{i=1}^{n_{c}} \boldsymbol{J}_{i}^{T} \boldsymbol{F}_{\boldsymbol{i}}+\left(\begin{array}{c}
\boldsymbol{\tau}_{\boldsymbol{\delta}, \text { base }} \\
\boldsymbol{\tau}_{\boldsymbol{\delta}, \text { joint }}
\end{array}\right) .
\end{aligned}
$$

In Eq. (2), we focus on the equation of the base link and estimate the undesired contact force acting on the base link as:

$$
\boldsymbol{\tau}_{\boldsymbol{\delta}, \text { base }}=-\sum_{i=1}^{n_{c}} \boldsymbol{J}_{i, \text { base }}^{T} \boldsymbol{F}_{\boldsymbol{i}}-\boldsymbol{\tau}_{\mathrm{dyn}}
$$

where $\boldsymbol{J}_{i \text {, base }}$ is a Jacobian of the force sensor link to the base link, $\tau_{\text {dyn }}$ is the left side of Eq. (2) at the base link.

In Eq. (3), $\boldsymbol{\tau}_{\delta \text {, base }}$ consists of the linear force part $\boldsymbol{\tau}_{\delta \text {, base }}^{v}$ and rotational torque part $\tau_{\delta \text {,base }}^{\omega}$. We assume that most contacts mainly apply the linear force to a robot and the torque part can be ignored as $\boldsymbol{\tau}_{\delta \text {, base }}^{\omega}=\mathbf{0}$. From the norm of the estimated contact force $\tau_{\delta \text {, base }}^{v}$ in Eq. (3), we can detect the contact by thresholding the force:

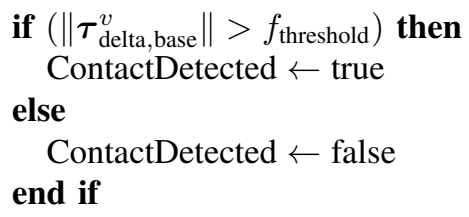

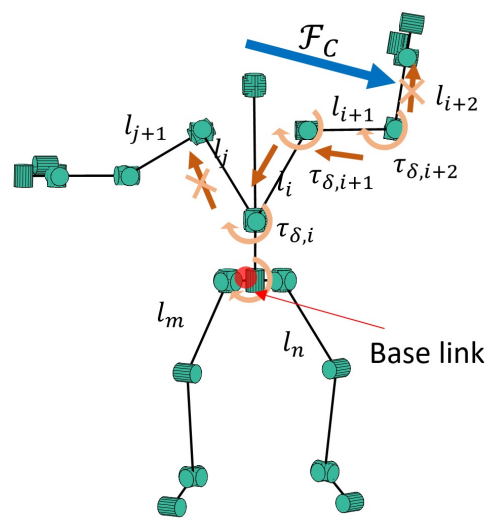

Fig. 1. Contact link searching algorithm. The contact force is applied to link $l_{i+2}$. The contact force generates the error torque $\tau_{i+2}$ at self-joint and every predecessor joint until base link but no error torque is generated at the links in another branches like link $l_{j}, l_{m}$ or $l_{n}$. Tracing the link containing the error torque, the contact link can be found.

$f_{\text {threshold }}$ is the threshold value to allow the errors due to a modeling error, sensor noises and the accuracy of sensors. We can choose a small threshold value for more sensitivity of the contact detection but it also becomes sensitive to the errors. On the other hand, we can choose a high threshold for more robust to the errors but it becomes less sensitive to the external force. In this paper, we practically set the value to $1.5 \%$ of the gravitational force due to the total mass of the robot.

After detecting the contact, we can simply estimate the contact force strength as $\tau_{\delta \text {,base }}^{v}$ Through the above procedure, we can detect the contact and identify the contact force strength at the same time.

\section{B. Contact link and point estimation}

Once contact is detected, we search the contact link by inspecting the error torque $\tau_{\delta, i}$ along the kinematic chain of the robot. The contact link searching is done by a simple depth-first search algorithm, which inspects whether the error torque is higher than the threshold from the base link to distal links. Fig. 1 shows the contact link searching algorithm. Since the successor joint torque cannot be affected by the predecessor joint torque, the link attached to the most distal joint containing the error torque is the link in contact. In Fig. 1 , let us assume that the contact force is applied to link $l_{i+2}$ the contact force generates the error torque $\tau_{i+2}$ at self-joint and every predecessor joint until the base link but no error torque is generated at the links in other branches like the link $l_{j}, l_{m}$ or $l_{n}$. When the contact link is found, the contact point can be estimated by computing the intersection between the convex hull or the mesh from the robot 3D model and the line of action of contact force.

When the contact force is applied to the contact link, both contact force and link are now known as a result of the previous step. Now, the external forces due to the contact force can be written with an unknown Jacobian to contact point.

$$
\tau_{\delta}=J_{C}^{T} F_{C}
$$


where $J_{C}^{T}$ is a Jacobian of the unknown contact point $x_{C}$ expressed in the local frame of its link, and $\boldsymbol{F}_{C}$ is the local frame representation of $\boldsymbol{\tau}_{\delta \text {,base }}^{v}\left(\boldsymbol{F}_{\boldsymbol{C}}=R_{c}^{T} \boldsymbol{\tau}_{\delta \text {,base }}^{v}\right)$.

In order to reconstruct the line of action, we can replace the unknown Jacobian $J_{C}$ on the contact link with known geometric Jacobian $J_{t, i}$ and $J_{r, i}$ for the linear and the rotational velocity respectively,

$$
J_{C}=J_{t, i}-\left[x_{C} \times\right] J_{r, i},
$$

where $[* x]$ denotes the skew-symmetric matrix representation instead of the cross product operation. From Eq. (4) and Eq. (5), we obtain the following equation,

$$
\begin{aligned}
\boldsymbol{\tau}_{\boldsymbol{\delta}} & =\left(\boldsymbol{J}_{\boldsymbol{t}, \boldsymbol{i}}-\left[\boldsymbol{x}_{\boldsymbol{C}} \times\right] \boldsymbol{J}_{\boldsymbol{r}, \boldsymbol{i}}\right)^{T} \boldsymbol{F}_{\boldsymbol{C}} . \\
& =\left(\boldsymbol{J}_{\boldsymbol{t}, \boldsymbol{i}} \boldsymbol{F}_{\boldsymbol{C}}-\boldsymbol{J}_{\boldsymbol{r}, \boldsymbol{i}}\right)^{T}\left[\boldsymbol{F}_{\boldsymbol{C}} \times\right] \boldsymbol{x}_{\boldsymbol{C}} .
\end{aligned}
$$

Although the contact point $x_{C}$ is unknown in Eq. (6), the line of action can be reconstructed by the following equation,

$$
\left[\boldsymbol{F}_{\boldsymbol{C}} \times\right] \boldsymbol{x}_{\boldsymbol{C}}=-\left(\boldsymbol{J}_{\boldsymbol{r}, \boldsymbol{i}}\right)^{\dagger}\left(\boldsymbol{F}_{\boldsymbol{C}}-\boldsymbol{J}_{\boldsymbol{t}, \boldsymbol{i}}\right)^{T} \boldsymbol{F}_{\boldsymbol{C}}
$$

where $\dagger$ denotes the pseudo-inverse operation. While Eq. (7) has three equations, only two equations are effective to compute the contact point $x_{C}$ due to rank deficit of the skewsymmetric matrix.

We use the triangular mesh generated from robot 3D CAD model to find the contact point as an intersection between the line of action and the triangular mesh ${ }^{2}$ (i.e. check the intersection of the action line with each triangle composing a given link). We then can have zero or multiple intersections. In the case of no intersection, the considered link is not in contact for the given link. In the case of the intersection(s), the contact point is found. If there are more than two, one of them is chosen by considering the robot posture. We can use the convex hull to avoid this conflict of multiple intersections yet, using the polygon mesh directly triangulated from the 3D CAD model gives more precise contact points.

The contact estimation method in this section is assessed on our humanoid robot in the next section.

\section{EXPerimental Result in Simulated AND ReAL HUMANOID ROBOT}

We implemented our contact estimation method on our humanoid robot HRP-4 to assess its feasibility. In this section, the contact detection and the contact point estimation on simulated humanoid is shown then the same procedure is ported on the real humanoid hardware, and the results are also shown. We apply a single contact force to the robot. Although our method can be applied to the dynamic situation, the dynamic case is left for future works and only the static case is shown in this paper.

${ }^{2}$ Clean triangular meshes can be obtained from points or polygons using CGAL library.

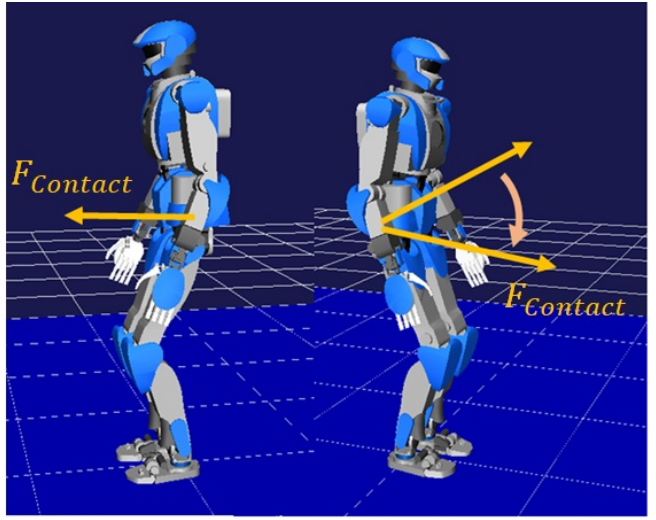

Fig. 2. Applying a single force to left and right hands in simulation. The force applied to left elbow link is fixed and the direction of the force applied to right arm is moving down.

\section{A. Contact Estimation in Simulation}

Before we implemented our contact estimator on the real robot, we have investigated its performance in simulation. In this experiment, we apply the force to a simulated humanoid and confirm whether the contact detection and point estimation can be correctly achieved.

The contact forces are applied to the left and right elbow link as shown in Fig. 2. The direction of the applied force at the left elbow link is fixed, whereas it is moving when applied at the right elbow link. The forces for both links are pulling forces, which contact points are fixed. The force strength is approximately 25 to $30 \mathrm{~N}$, which is applied continuously during $8 \mathrm{sec}$ and $18 \mathrm{sec}$ respectively.

The result of the experiment in simulation is shown in Fig. 3 with identified contact force strength that is the norm of contact force in Eq. (3) (left $y$-axis) and contact detected link index (right $y$-axis). According to the result, the contact is detected at the left elbow link indicated as link index No. 30 in Fig. 3 from $2 \mathrm{sec}$ to $12 \mathrm{sec}$ then it is detected at the right elbow link (link index No. 21) from $22 \mathrm{sec}$ to $40 \mathrm{sec}$.

When the contact link is detected, a line of action of the contact force is reconstructed and used to compute the contact point. The latter is the result of the intersection between the reconstructed line of action and the robot's link polygon meshes. The reconstruction of the line is derived by Eq. (7) in Section II-B. For generating the polygon meshes of robot links, we used the 3D point set of the CAD model. The outcome is a list of triangles, thus, the contact points are computed as intersections between lines and each triangle. As a result, we can have one or more points pulling (force vector goes out from a robot body) and pushing away (force vector goes into a robot body). In case where more than two contact points computed, we can chose one of them, considering the task the robot is doing or the environment surrounding the robot, otherwise, we chose the closest one to the initial point of the reconstructed line (segment) because most of contact force vector is pushing away unless the body is hung on something.

Fig. 4 shows the body mesh, reconstructed lines and contact points marked on the body surface. Contact points are 

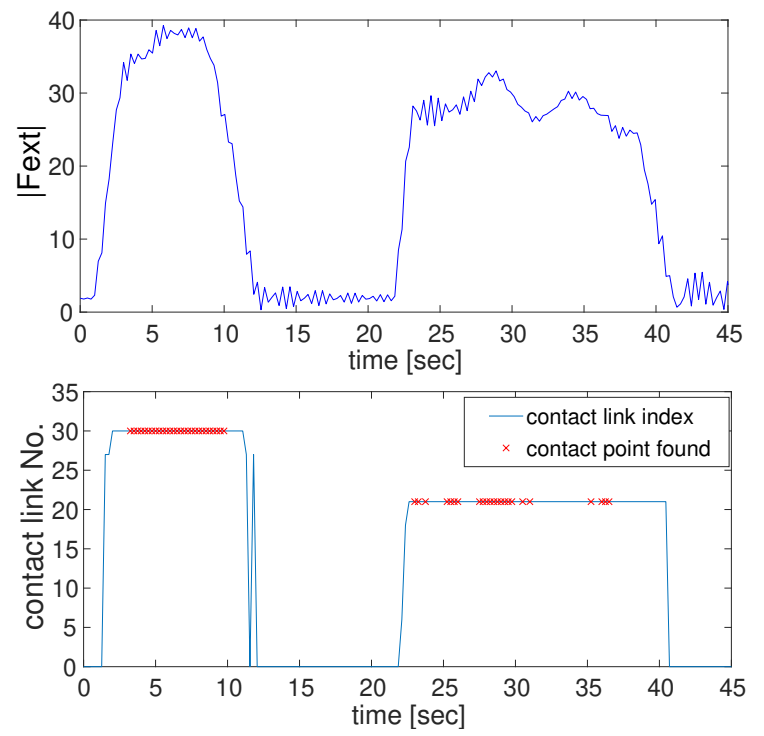

Fig. 3. Result of contact link detection, force strength identification and contact point estimation in simulation. Upper part indicates force strength of contact force and lower part indicates the contact link index (No. 21 is right elbow link, No. 30 is left elbow link).

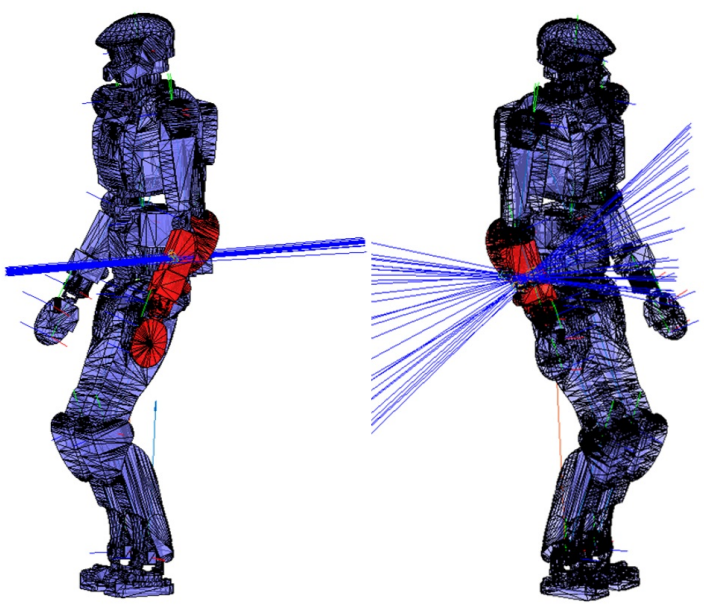

Fig. 4. Reconstructing a line of action of contact forces and computing the intersections between a line and the robot body mesh. Right side figure shows the result of the left elbow link and the left side shows that of the right one. The detected contact links are highlighted in red.

not always found due to the accuracy of the reconstructed line of action. Each line is depicted at $250 \mathrm{~ms}$ intervals. In Fig. 3, read marks " $x$ " are placed when the contact points are found. During $3 \mathrm{sec}$ to $8 \mathrm{sec}$ in Fig. 3, the reconstructed lines are stable and always going across a mesh, therefore, the contact points are correctly computed on the elbow link surface. In the case of right arm during $22 \mathrm{sec}$ to $40 \mathrm{sec}$, the lines are reconstructed however, some of them are not going across a mesh, which is caused by a mismatch of the reconstructed line. Even in the case where the contact link detection is correctly done, the contact point estimation may have failed due to inaccuracy in the robot state estimation. Even in simulation, contact forces resulting from virtual pushes may have numerical issues sometimes and this may cause such failures. This problem is discussed in the experiments conducted on the real humanoid (see next section). In both cases, the contact link is detected and the direction of the line of action is correctly reconstructed.

\section{B. Contact Estimation on the Real Humanoid Robot and Practical Discussions}

We apply the same method to the real humanoid robot setup to assess both the feasibility and performance of the proposed estimator on real hardware. Comparing the real robot with the simulated one, there are many differences such as the sensor noise, the joint flexibility and the friction problems. Thus, how these problems affect the estimator is discussed at the end of this section.

We use the human-sized humanoid robot HRP-4 that height and weight is $155 \mathrm{~cm}$ and $40 \mathrm{~kg}$ respectively. The robot has 9 degrees of freedom (dofs) (shoulder: 3 dofs, elbow: 1, wrist: 3, hand: 2) in each arm, 7 dofs in each leg (hip: 3, knee: 1, ankle: 2), 3 dofs in chest, 2 dofs in neck and total dofs is 37 . HRP-4 is position-based controlled robot with high PD gains; it has an accelerometer and gyroscope mounted at the base link for its state estimation, encoders mounted at each joint for joint angles $\boldsymbol{q}$ and joint angular velocity $\dot{\boldsymbol{q}}$, current sensors mounted at each joint for joint torque $\tau_{m}, 6$-axis force/torque sensor mounted at each foot link for floor reaction force.

In this experiment, we apply a single force to the right elbow link (Fig. 5(a).), right shoulder link (Fig. 5(b).), left elbow link (Fig. 5(c).), left shoulder link (Fig. 5(d).) in this order. Fig. 5 depicts the position where the force is applied and the direction of the force. The direction of the applied force to right and left elbow links is almost similar to the direction of the applied force to right and left shoulder links as shown in Fig. 5. During the experiment, we measured the applied force strength by a 1-axis force gauge so that we can compare it with the estimated force.

Fig. 6 shows the result of contact link detection and force identification. The estimated contact force is shown with a solid-line and the measured contact force by force gauge is drawn with a dashed-line. In Fig. 6, first, we apply the force to the right elbow link (Fig. 5(a).) although the shoulder link is detected. One of the reasons that the contact link is mismatched with the expected one is considered due to the joint friction or high stiffness due to a high proportional gain for the feedback control. Because the gear ratio of the elbow joint is very high, the static friction is therefore also high, reducing the torque caused by the contact force. As a result, the contact is detected at the shoulder link that is the predecessor link of the elbow link. Although the contact detection failed, the contact force is successfully estimated. The contact link detection and contact force estimation are independent in the way they are processed. Thus, there are cases where one of them is correct even if the other is not. Next, about $30 \mathrm{~N}$ of the force is applied to the right shoulder link (Fig. 5(b).) at around $30 \mathrm{sec}$ in Fig. 6. In this period, the contact link detection succeeded, however, the estimated contact force has a bias error by about $7 \mathrm{~N}$. Another force is then applied to the left elbow link (Fig. 5(c).) but the left shoulder link is detected, similarly to the right elbow 


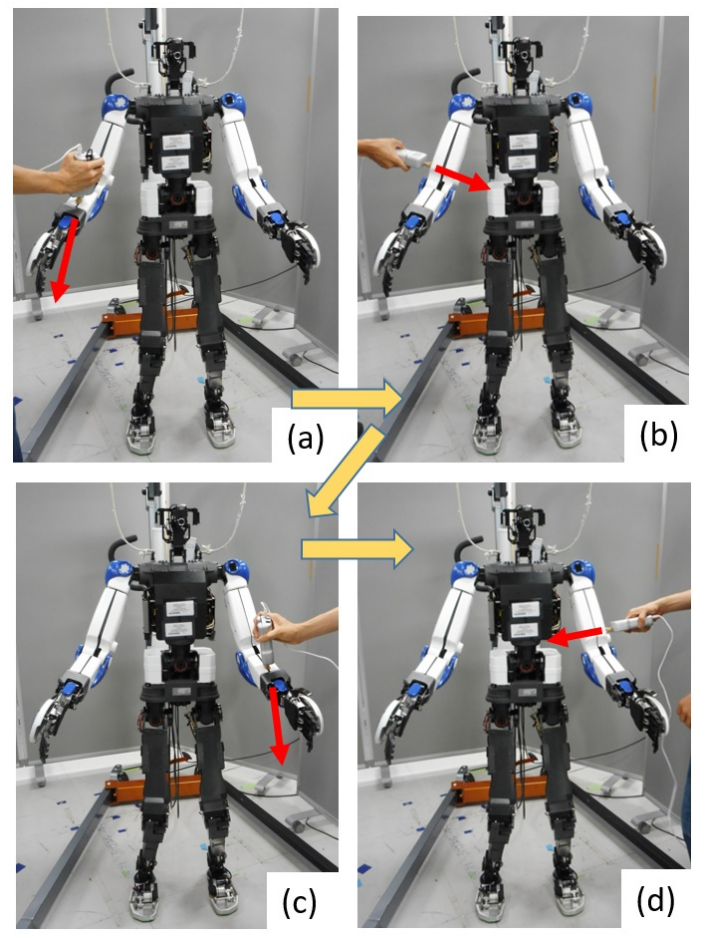

Fig. 5. Applying a single force to right and left arms in HRP-4. The force was applied to right elbow link (a), right shoulder link (b) then, left elbow link (c), left shoulder link (d) by fixed direction.

case. The contact force estimation succeeded as its strength is similar to the measured one. Finally, we apply the force to the left shoulder (Fig. 5(d).). Similarly to the right link case, the contact link detection succeeded. However, we observed a difference of approximately $5 \mathrm{~N}$ between the estimated and measured force.

In the real robot experiment, the contact point is not found due to the incorrect link detection or the inaccuracy of the error torque $\tau_{\delta}$ at each joint. Even in the case where the estimator detects an incorrect link, if that link is the predecessor link of the correct one, it might be possible to find a contact point by using the mesh associated with that of the successor link. For instance, in the previous experimental result, using the joined body mesh of the right shoulder link and elbow link instead of using the single body mesh of the right shoulder might allow to find the contact point. Therefore, it is better to use all links of a given tree to choose the best match. In Fig. 7, the joined body mesh of left shoulder and elbow link is shown in red mesh. Some of the reconstructed lines are going across the joined body mesh, which gives the intersections as a result. Determining the threshold for each joint based upon the joint stiffness instead of using is unique value is one of the possible solutions for avoiding misdetection.

\section{CONCLUSIONS}

We proposed a contact estimation method for a humanoid robot that allows to identify the contact force strength, detect the contact link and estimate the contact point by using the meshes of the robot body. The contact force identification can be performed by computing the error between the generalized
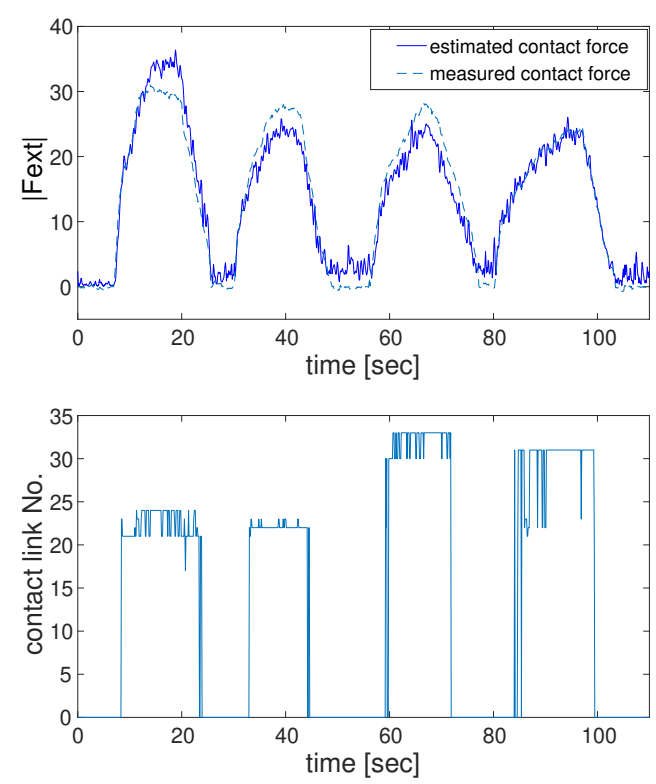

Fig. 6. Result of contact link detection, force strength identification and contact point estimation in HRP-4. Left $y$-axis indicates force strength of contact force and right $y$-axis indicates the contact link index (No. 21-23 is right shoulder link, No. 24 is right elbow link, No. 30-32 is left shoulder link and No. 33 is left elbow link).

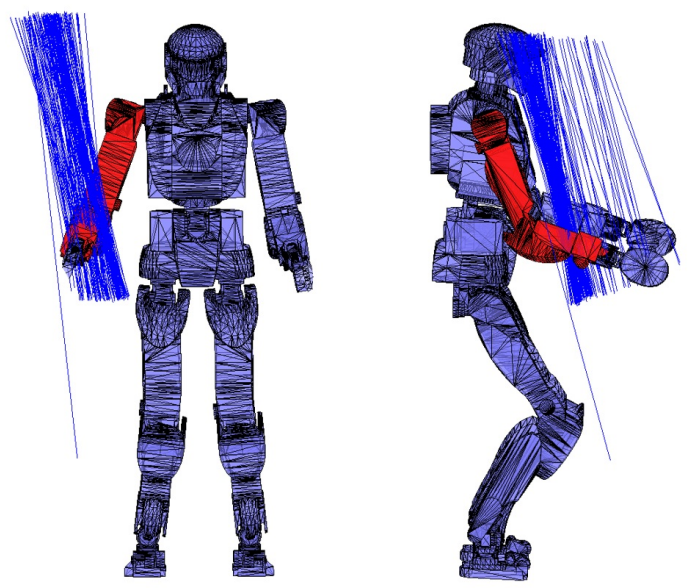

Fig. 7. Reconstructing a line of action of contact forces and computing the intersections between a line and the joined body mesh. The joined body surface of left shoulder link and elbow link are shown in red. Right side figure shows the result of left link and left side shows that of right one.

forces estimated from the expected robot state and those measured from the real robot sensory data. The estimator can basically provide the resultant forces which allow to detect reliably single contact force. Extension to multiple contact forces is possible by tracing the joint torques of all tree and achieving the total wrench force distribution weighted by the excess of torques. For the contact link detection, the contact link is similarly derived by tree search method using robot kinematic chains. Finally, after reconstructing the line of action of the contact force, the contact point is estimated as the intersection between the reconstructed line and the triangles composing the mesh of the link or its convex hull.

The contact point estimation succeeded when applying the external force with almost time-constant force strength 
value and fixed direction; on the other hand, it failed when applying the force with time-varying strength value and direction. This issue needs to be investigated further. In the real robot experiment, the accuracy of the estimated contact force strength was depending on the link where the external force is applied, or on the direction of the applied force. The contact point estimation is not perfect on the real robot, which may be caused by the following two different issues. One of the two is that misdetection of the contact link. In this case, obviously, the intersection cannot be found because the line does not cross the link's mesh. The other issue is the inaccuracy of the estimated error torque produced by the contact force. As the error is computed from the inverse dynamics computation of the robot model, the latter problem is due to the modeling error about the inertial parameter of the robot. However, this error can be improved by a reidentification of the inertial parameter [25], [26]. Adding the joint friction model to the robot dynamics equation also might improve robustness and precision. Those issues will be considered in our future works, together with the extension of our method to the multiple contact situation and the dynamic motion case.

\section{ACKNOWLEDGMENT}

The authors would like to express sincere gratitude to Kevin Chappellet of LIRMM for his technical support and advice for the experiment and acknowledge the student exchange program of the French Embassy at Tokyo, Japan.

\section{REFERENCES}

[1] Ko Ayusawa, Eiichi Yoshida, Yumeko Imamura, and Takayuki Tanaka, "New evaluation framework for human-assistive devices based on humanoid robotics," Advanced Robotics, vol. 30, no. 8, pp. 519-534, 2016.

[2] Cota Nabeshima, Ko Ayusawa, Conrad Hochberg, and Eiichi Yoshida, "Standard performance test of wearable robots for lumbar support," IEEE Robotics and Automation Letters, vol. 3, no. 3, pp. 2182-2189, 2018.

[3] Takahiro Ito, Eiichi Yoshida, Ko Ayusawa, and Hiroshi Kobayashi, "Stationary torque replacement for evaluation of active assistive devices using humanoid," in Proc. 2016 IEEE-RAS Int. Conf. on Humanoid Robots, 11 2016, pp. 739-744.

[4] Aiman Musa M Omer, Hideki Kondo, Hun-ok Lim, and Atsuo Takanishi, "Development of walking support system based on dynamic simulation," in Robotics and Biomimetics, 2008. ROBIO 2008. IEEE International Conference on. IEEE, 2009, pp. 137-142.

[5] Kanako Miura, Eiichi Yoshida, Yoshiyuki Kobayashi, Yui Endo, Fumio Kanehiro, Keiko Homma, Isamu Kajitani, Yoshio Matsumoto, and Takayuki Tanaka, "Humanoid robot as an evaluator of assistive devices," in Proc. 2013 IEEE Int Conf. Robotics and Automation, 2013, pp. 671-677.

[6] Luis Sentis, Jaeheung Park, and Oussama Khatib, "Compliant control of multicontact and center-of-mass behaviors in humanoid robots," IEEE Transactions on robotics, vol. 26, no. 3, pp. 483-501, 2010.

[7] Hervé Audren, Joris Vaillant, Abderrahmane Kheddar, Adrien Escande, Kenji Kaneko, and Eiichi Yoshida, "Model preview control in multi-contact motion-application to a humanoid robot," in Intelligent Robots and Systems (IROS 2014), 2014 IEEE/RSJ International Conference on. IEEE, 2014, pp. 4030-4035.

[8] Tommaso Mattioli and Marilena Vendittelli, "Interaction force reconstruction for humanoid robots," IEEE Robotics and Automation Letters, vol. 2, no. 1, pp. 282-289, 2017.

[9] Fabrizio Flacco, Antonio Paolillo, and Abderrahmane Kheddar, "Residual-based contacts estimation for humanoid robots," in $\mathrm{Hu}$ manoid Robots (Humanoids), 2016 IEEE-RAS 16th International Conference on. IEEE, 2016, pp. 409-415.
[10] Jonathan Vorndamme, Moritz Schappler, and Sami Haddadin, "Collision detection, isolation and identification for humanoids," in Robotics and Automation (ICRA), 2017 IEEE International Conference on. IEEE, 2017, pp. 4754-4761.

[11] Salman Faraji and Auke Jan Ijspeert0, "Designing a virtual whole body tactile sensor suit for a simulated humanoid robot using inverse dyanmics," in 2016 IEEE/RSJ Int. Conf. on Intelligent Robots and Systems (IROS), 10 2016, pp. 5564-5571.

[12] Anastasia Bolotnikova, Sébastien Courtois, and Abderrahmane Kheddar, "Contact observer for humanoid robot pepper based on tracking joint position discrepancies," in IEEE International Symposium on Robot and Human Interactive Communication, Nanjing, China, 27-31 August 2018, pp. 29-34.

[13] Samuel Agus Setiawan, Jin'ichi Yamaguchi, Sang Ho Hyon, and Atsuo Takanishi, "Physical interaction between human and a bipedal humanoid robot-realization of human-follow walking," in Robotics and Automation, 1999. Proceedings. 1999 IEEE International Conference on. IEEE, 1999, vol. 1, pp. 361-367.

[14] Kazuhiko Yokoyama, Hiroyuki Handa, Takakatsu Isozumi, Yutaro Fukase, Kenji Kaneko, Fumio Kanehiro, Yoshihiro Kawai, Fumiaki Tomita, and Hirohisa Hirukawa, "Cooperative works by a human and a humanoid robot," in IEEE International Conference on Robotics and Automation. IEEE; 1999, 2003, vol. 3, pp. 2985-2991.

[15] Sang-Ho Hyon, Joshua G Hale, Gordon Cheng, et al., "Full-body compliant human-humanoid interaction: Balancing in the presence of unknown external forces," IEEE Trans. Robotics, vol. 23, no. 5, pp. 884-898, 2007.

[16] Anastasia Bolotnikova, Sébastien Courtois, and Abderrahmane Kheddar, "Compliant motion of a humanoid robot regulated via proprioceptive sensor based contact observer," in IEEE-RAS International Conference on Humanoid Robots, Beijing, China, 6-9 November 2018.

[17] Takahiro Ito, Ko Ayusawa, Eiichi Yoshida, and Hiroshi Kobayashi, "Human motion reproduction by torque-based humanoid tracking control for active assistive device evaluation," in Humanoid Robotics (Humanoids), 2017 IEEE-RAS 17th International Conference on. IEEE, 2017, pp. 503-508.

[18] Johannes Englsberger, Alexander Werner, Christian Ott, Bernd Henze, Maximo A Roa, Gianluca Garofalo, Robert Burger, Alexander Beyer, Oliver Eiberger, Korbinian Schmid, et al., "Overview of the torquecontrolled humanoid robot toro," in Humanoid Robots (Humanoids), 2014 14th IEEE-RAS International Conference on. IEEE, 2014, pp. 916-923.

[19] Nicolaus A Radford, Philip Strawser, Kimberly Hambuchen, Joshua S Mehling, William K Verdeyen, A Stuart Donnan, James Holley, Jairo Sanchez, Vienny Nguyen, Lyndon Bridgwater, et al., "Valkyrie: Nasa's first bipedal humanoid robot," Journal of Field Robotics, vol. 32, no. 3, pp. 397-419, 2015.

[20] Christian Ott, Bernd Henze, and Dongheui Lee, "Kinesthetic teaching of humanoid motion based on whole-body compliance control with interaction-aware balancing," in Proceedings of the IEEE/RSJ International Conference on Intelligent Robots and Systems (IROS), 2013, pp. 4615-4621.

[21] Alessandro De Luca, Alin Albu-Schaffer, Sami Haddadin, and Gerd Hirzinger, "Collision detection and safe reaction with the dlr-iii lightweight manipulator arm," in Intelligent Robots and Systems, 2006 IEEE/RSJ International Conference on. IEEE, 2006, pp. 1623-1630.

[22] Alessandro De Luca and Fabrizio Flacco, "Integrated control for phri: Collision avoidance, detection, reaction and collaboration," in Biomedical Robotics and Biomechatronics (BioRob), 2012 4th IEEE RAS \& EMBS International Conference on. IEEE, 2012, pp. 288-295.

[23] Karim Bouyarmane, Kevin Chappellet, Joris Vaillant, and Abderrahmane Kheddar, "Multi-robot and task-space force control with quadratic programming," IEEE Transactions on Robotics, 2019, to appear.

[24] Kenji Kaneko, Fumio Kanehiro, Mitsuhari Morisawa, Kazuhiko Akachi, Go Miyamori, Atsushi Hayashi, and Noriyuki Kanehira, "Humanoid robot HRP-4 - humanoid robotics platform with lightweight and slim body," in Proc. 2011 IEEE/RSJ Int. Conf. on Intelligent Robots and Systems, 2011, pp. 4400-4407.

[25] W. Khalil and E. Dombre, Modeling, identification and control of robots, Hermès Penton, London-U.K, 2002.

[26] K. Ayusawa, G. Venture, and Y. Nakamura, "Identifiability and identification of inertial parameters using the underactuated base-link dynamics for legged multibody systems," Int. J. of Robotics Research, vol. 33, no. 3, pp. 446-468, 2014. 